\title{
Spin-polarized ballistic transport in a thin superlattice of zinc blende half-metallic compounds
}

\author{
M. C. Qian, ${ }^{1, *}$ C. Y. Fong, ${ }^{1}$ W. E. Pickett, ${ }^{1}$ J. E. Pask, ${ }^{2}$ L. H. Yang, ${ }^{2}$ and S. Dag ${ }^{3}$ \\ ${ }^{1}$ Department of Physics, University of California, Davis, California 95616-8677, USA \\ ${ }^{2}$ H Division, Lawrence Livermore National Laboratory, Livermore, California 94551, USA \\ ${ }^{3}$ Department of Physics, Bilkent University, Ankara 06800, Turkey
}

(Received 23 September 2004; published 24 January 2005)

\begin{abstract}
We examine theoretically ballistic conduction in thin layers of zinc blende half metals, considering as an example a superlattice consisting of monolayers of GaAs and MnAs, a bilayer of CrAs, and a bilayer of GaAs. By artificially separating bilayers, we show that surface states thwart half metallicity. However, capping the metal-As bilayers restores half metallicity, and ballistic conduction of electrons within $\sim 0.3 \mathrm{eV}$ of the Fermi level will give nearly $100 \%$ spin-polarized transmission in the direction of the superlattice. Recent developments suggest atomic layer epitaxy can be used to produce such thin layers for spintronic applications.
\end{abstract}

DOI: $10.1103 /$ PhysRevB.71.012414

PACS number(s): 75.50.Pp, 75.70.Cn, 71.20.-b, 73.50.-h

Since their discovery by de Groot et al. ${ }^{1}$ in 1983 , half metallic (HM) compounds, ${ }^{2}$ in which one spin channel is metallic while the other is semiconducting, have been the focus of much research. ${ }^{3-8}$ The interest in these novel materials is practical as well as fundamental, as the $100 \%$ spin polarization at the Fermi energy offers potentially significant advantages for spintronic device applications, ${ }^{9-11}$ in which the spin of electrons is exploited as well as the charge.

After the pioneering work of Akinaga et $a .^{3}$ predicting the half metallic behavior of zinc blende (ZB) CrAs and subsequently growing it in thin film form, several such transition metal compounds have been investigated experimentally ${ }^{4}$ and theoretically. ${ }^{5-8}$ In all HM ZB transition metal pnictides, the majority spin channel is metallic while the minority channel is semiconducting. ${ }^{8}$ To search for new HM materials based on these ZB-HMs, theoretical studies of various heterostructures ${ }^{5,12}$ have been carried out. Sanvito and Hill ${ }^{5}$ have examined the electronic and transport properties of a digital ferromagnetic heterostructure ${ }^{13}$ composed of a monolayer of MnAs and 16 layers of GaAs. They found that the in-plane conduction is confined to the vicinity of the MnAs layer. Experimentally, it has been found that bilayer CrAs and GaAs can be grown in the ZB structure to form a CrAs/GaAs superlattice by molecular beam epitaxy. ${ }^{14}$ Furthermore, ZB MnAs monolayers ${ }^{13}$ have been successfully embedded into thick GaAs. Therefore, it is of interest to investigate how a thin film superlattice involving these HMs can exhibit half metallic properties and ballistic transport in the direction of growth.

In this paper, we address these issues using a thin superlattice composed of MnAs, CrAs, and GaAs, by carrying out first-principles calculations. We start with a model consisting of three separate regions: a region containing a monolayer of GaAs and a monolayer of MnAs, which we denote by $\mathrm{Ga}_{0.5} \mathrm{Mn}_{0.5} \mathrm{As}$, a region containing a bilayer of CrAs, and a region containing a bilayer of GaAs. Then we bring them together to form a model superlattice and address the question of ballistic conduction by examining the density and dispersion of states in the vicinity of the Fermi energy $E_{F}$. We find that the superlattice is half metallic, with $100 \%$ spinpolarized ballistic transport in the growth direction.

First-principles total energy electronic-structure calcula- tions have been performed using the plane wave pseudopotential method ${ }^{15}$ based on density functional theory ${ }^{16}$ in the generalized gradient approximation (GGA) ${ }^{17} \mathrm{We}$ used the VASP implementation ${ }^{18,19}$ with ultrasoft pseudopotentials ${ }^{20}$ for all atoms. Spin polarized calculations were carried out to account for different spin channels. A plane wave cutoff of $450 \mathrm{eV}$ was used, and an $11 \times 11 \times 7$ Monkhorst-Pack ${ }^{21}$ k-point mesh was employed, corresponding to $144 \mathbf{k}$-points in the irreducible Brillouin zone. Tests with larger $\mathbf{k}$ point sets yielded changes in total energy of less than $1 \mathrm{meV}$.

To investigate the formation of a conducting channel, we started with a model in which $\mathrm{Ga}_{0.5} \mathrm{Mn}_{0.5} \mathrm{As}$, CrAs, and GaAs are separated into three slab regions [Fig. 1(a)]. We then moved the regions together until all atoms were at GaAs bulk positions [Fig. 1(b)]. The GGA optimized lattice constant of GaAs $\left(a_{0}=5.722 \AA\right)$ was used for all calculations. Sectional views of the superlattice are shown in Fig. 1. The unit cell is tetragonal with lattice constant $a_{0} / \sqrt{2}$ in the a and b directions. Figure 1(a) shows the slab configuration with

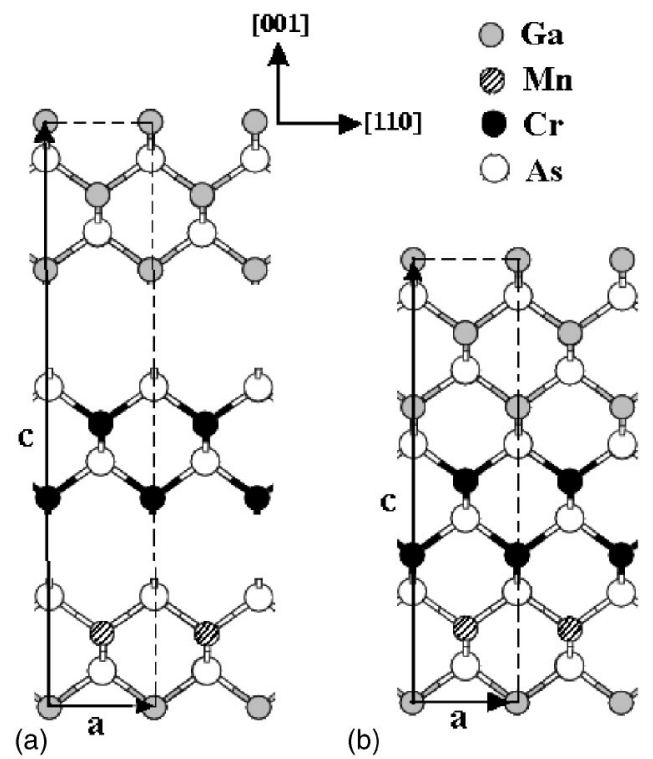

FIG. 1. (a) Separated and (b) connected superlattice, viewed along $\mathbf{b}$. $\mathbf{b}$ is the $[\overline{\mathbf{1 1 0}}]$ direction. 

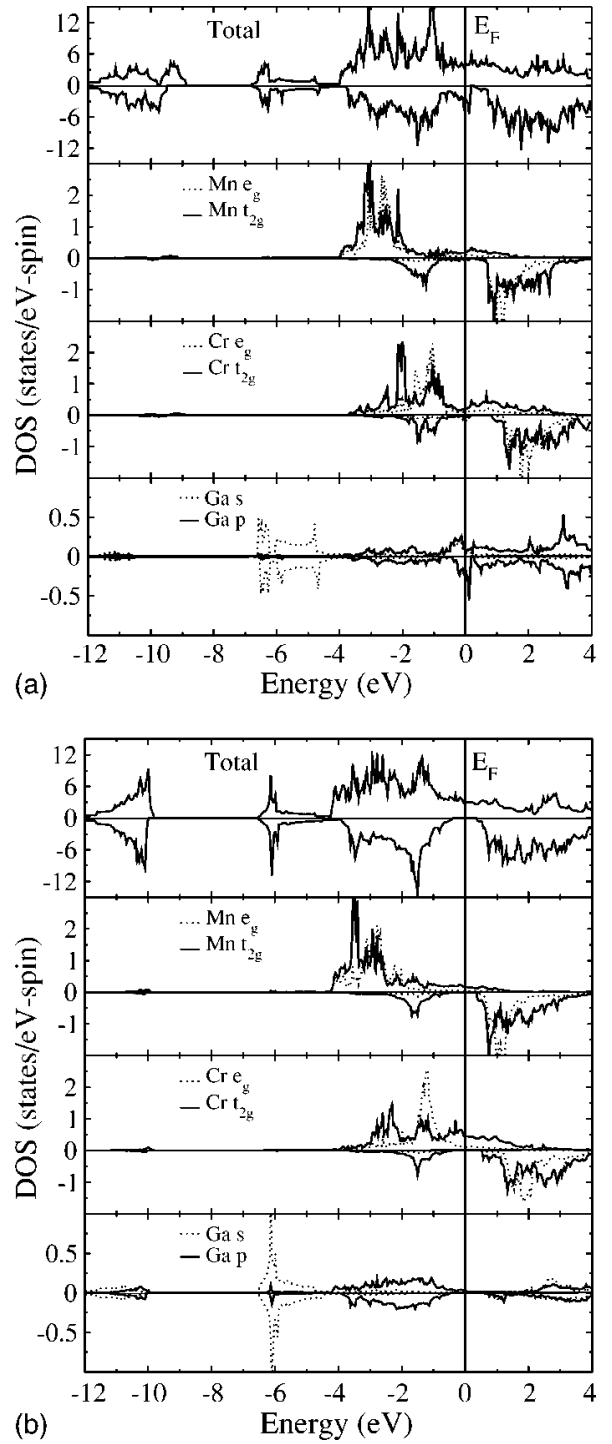

FIG. 2. Total and projected density of states of $\left(\mathrm{Ga}_{0.5} \mathrm{Mn}_{0.5} \mathrm{As}\right)(\mathrm{CrAs})(\mathrm{GaAs})$ for the thin super-lattice: (a) $c=4 a_{0}$ case; (b) $c=3 a_{0}$ case. The material is metallic. The positive (negative) values represent majority (minority) spin. $E_{F}=0$.

$c=4 a_{0}$ and Fig. 1(b) shows the superlattice with $c=3 a_{0}$. By reducing the spacing between the regions from the $4 a_{0}$ case to the $3 a_{0}$ case, we examine the effect of the surfaces on the electronic structure and the formation of a conducting channel in real space.

In the $c=4 a_{0}$ case, the system is a two-dimensional ferromagnetic metal with a net magnetic moment of $9.11 \mu_{B}$ per unit cell. Calculated total and projected densities of states (DOSs) are shown in Fig. 2(a). Both majority and minority DOSs are nonzero at $E_{F}$. Considering first the total DOS, we find low-lying, isolated As- $s$ states at $\sim-10 \mathrm{eV}, \mathrm{Ga}-s$ and As- $p$ bonding states at $\sim-6 \mathrm{eV}$, and metal- $d$-As- $p$ hybrid states in the vicinity of $E_{F}$. As shown in the projected DOSs panels, the Mn $d$ states are located lower in energy than the corresponding $\mathrm{Cr} d$ states, consistent with nuclear charges. Due to the lack of fourfold coordination, the surface $\mathrm{Ga}$ and $\mathrm{Cr}$ atoms contribute minority spin states at $E_{F}$ which destroy half metallicity (see $c=3 a_{0}$ discussion below). The total
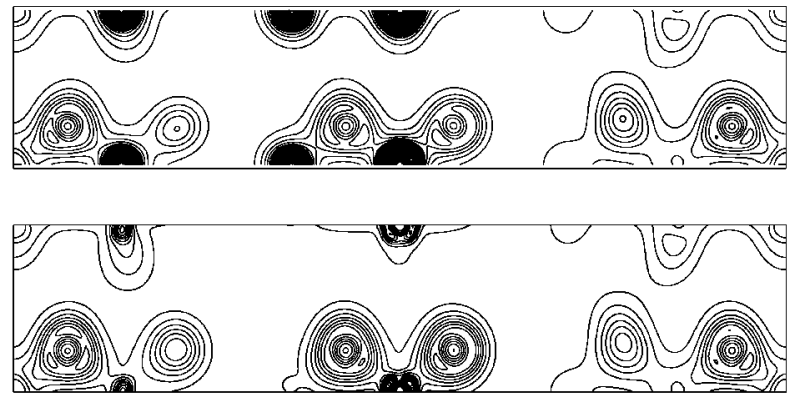

(a)
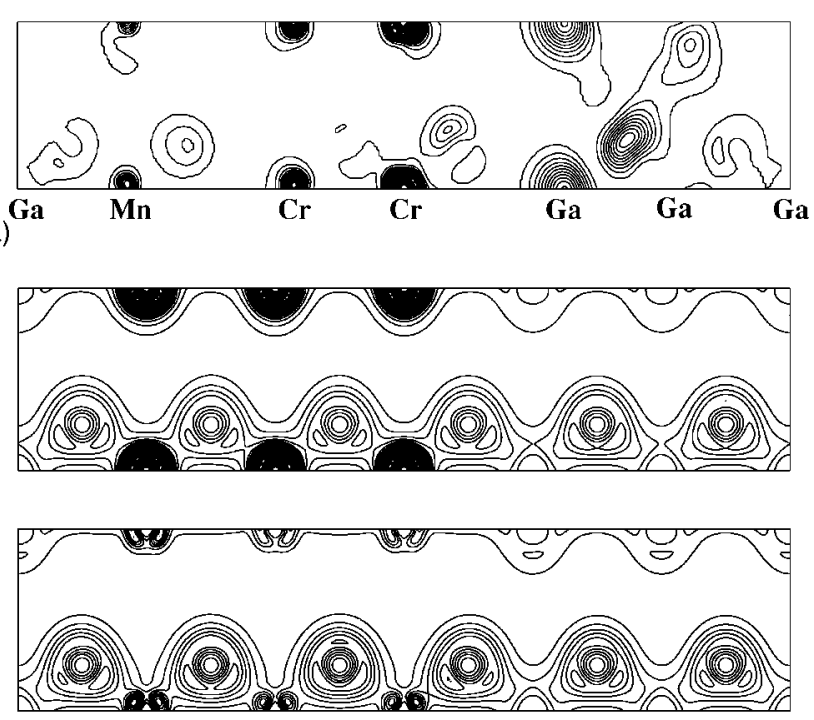

(b)

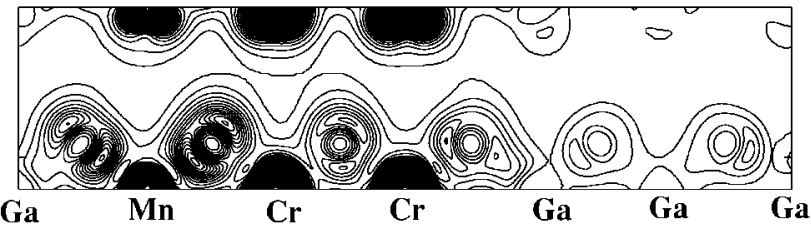

FIG. 3. Calculated charge densities in the $(0 \overline{1} 1)$ plane for the thin superlattice: (a) $c=4 a_{0}$ case; (b) $c=3 a_{0}$ case. The upper, middle, and bottom panels represent the majority spin channel, minority spin channel, and conducting states with energies $\sim 0.3 \mathrm{eV}$ above $E_{F}$. The corresponding maximum values are 2.21, 0.47, and 0.03 electrons $/ \AA^{3}$ for $c=4 a_{0}$ case, and 2.93, 0.53, and 0.37 electrons $/ \AA^{3}$ for $c=3 a_{0}$ case, respectively. The contours are equally spaced between the maximum value and zero.

charge densities for the majority and minority states in a (011) plane containing the Ga-As-Mn-As, Cr-As-Cr-As, and Ga-As-Ga-As chains are shown in the upper and middle panels of Fig. 3(a), respectively. The indices are defined with respect to the conventional cell. There is essentially no overlap of charge between the regions. The calculated charge density for the states with energies $\sim 0.3 \mathrm{eV}$ above $E_{F}$ in the (011) plane is shown in the bottom panel of Fig. 3(a). These states can accommodate externally injected electrons. However, the charge distributions are confined in the three regions because of the energy barriers (vacuum) between them. No channel is formed along the $\mathbf{c}$ direction and conduction will be inhibited.

In the $c=3 a_{0}$ case, all atoms are fourfold coordinated and 
there are no surface states. This results in a gap in the minority channel at $E_{F}$ and so produces a HM structure. The magnetic moment is $10 \mu_{B}$ per unit cell, consistent with the moment equation of Ref. 12 for such composite structures. The saturation magnetization of the superlattice is consistent with that of the constituent compounds. ${ }^{12}$ The semiconducting gap in the minority channel is $0.95 \mathrm{eV}$, only slightly less than those of the constituent CrAs and MnAs compounds. To understand the electronic properties of the $c=3 a_{0}$ case, we examine total and projected DOSs in Fig. 2(b). As in the constituent ZB magnetic compounds, ${ }^{8}$ and the $c=4 a_{0}$ configuration above, we find isolated, low-lying As $s$ states at $\sim-10.0 \mathrm{eV}$. Metal- $d$-As- $p$ hybrid states appear $\sim 4 \mathrm{eV}$ below $E_{F}$. In the majority spin channel, the states at $-3.7 \mathrm{eV}$ show significant $\mathrm{Mn} t_{2 g}$ character while those at $-2.9 \mathrm{eV}$ are more strongly $\mathrm{Mn} e_{g}$ in nature. The broad manifold around $-2.0 \mathrm{eV}$ of strong $\mathrm{Cr} t_{2 g}$ character is associated with hybridized $\mathrm{Cr}-t_{2 g}-\mathrm{As}-p$ bonding states while the more localized manifold around $-1.2 \mathrm{eV}$ is associated with nonbonding $\mathrm{Cr}$ $e_{g}$ states. The minority channel shows similar structures but with $d$ states shifted upward in energy relative to the corresponding majority states by the exchange interaction. Because the more localized nonbonding $e_{g}$ states are shifted more than the strongly hybridized bonding $t_{2 g}$ states, a semiconducting gap is opened in the minority channel at $E_{F}$.

To examine the formation of a conducting channel in the $c=3 a_{0}$ configuration, we need only focus on the majority spin states since the minority spin channel is semiconducting with a $0.95 \mathrm{eV}$ gap. The calculated total charge densities in a (011) plane for the majority and the minority spin states are shown in the upper and middle panels of Fig. 3(b), respectively. The majority charge densities show metal- $d$-As- $p$ bonding and strongly localized $e_{g}$ densities at the metal atoms, consistent with the strong $p-t_{2 g}$ hybridization and nonbonding $e_{g}$ character evident in the projected DOS [Fig. 2(b)]. The minority charge densities also show the metal$d$-As- $p$ bonding with clear $t_{2 g}$ character at the metal atoms. The charge densities around the Ga atoms are essentially non-spin-polarized. The bottom panel of Fig. 3(b) shows the calculated charge distribution for majority spin states with energies $\sim 0.3 \mathrm{eV}$ above $E_{F}$. Along the zigzag chain in the (011) plane there are contours extending from the left end to the right end corresponding to a clear conducting channel, in marked contrast to the $c=4 a_{0}$ case. Figure 4 shows the calculated band structures for both cases. $\Gamma-Z$ is in the $\mathbf{c}$ direction, perpendicular to the layers. The $c=3 a_{0}$ majority spin bands in the vicinity of $E_{F}$ show significant dispersion whereas the corresponding $c=4 a_{0}$ bands are essentially flat. Thus the $c=3 a_{0}$ states not only form a clear conducting channel along the c direction but also show significant velocity components along that direction. The projection of the Fermi surface perpendicular to the $\mathbf{c}$ direction will then be substantially larger for the $c=3 a_{0}$ configuration, yielding a correspondingly larger ballistic conductance in that direction. Moreover, due to the half metallic nature of the $c=3 a_{0}$ superlattice, the current will be $100 \%$ spin polarized.

The ballistic conductance in the $\mathbf{c}$ direction can be calculated once the electronic wave function and energy are known: ${ }^{22}$

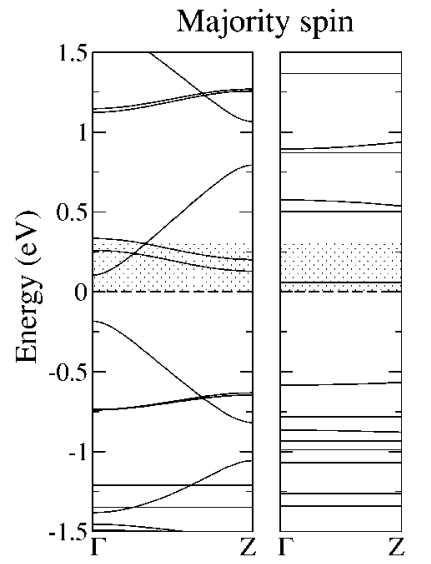

(a)

(b)

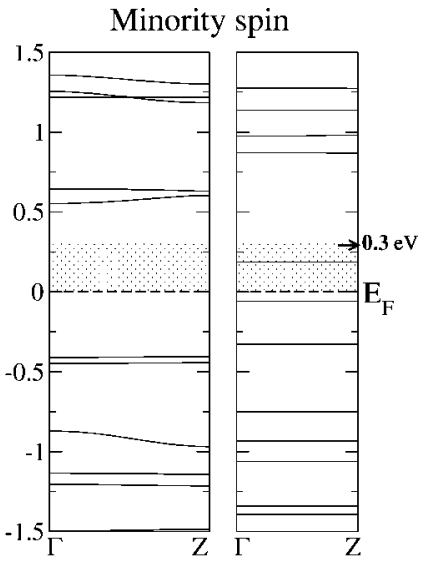

(a)

(b)
FIG. 4. Band structures along the $\mathbf{c}$ direction for the thin superlattice: (a) $c=3 a_{0}$ case; (b) $c=4 a_{0}$ case. The states with energies within $\sim 0.3 \mathrm{eV}$ of $E_{F}$ are shaded. $E_{F}=0$.

$$
G_{\sigma}=\frac{A e^{2}}{2 m} \sum_{\nu} \int \frac{d \mathbf{k}}{8 \pi^{3}}\left\langle\Psi_{\mathbf{k} \nu}\left|\mathbf{P}_{z}\right| \Psi_{\mathbf{k} \nu}\right\rangle \delta\left(\varepsilon_{\mathbf{k} \nu}-E_{F}\right)
$$

where $A$ is the finite cross section; $\mathrm{P}_{z}$ is the momentum operator, $\mathrm{P}_{z}=-i \hbar \nabla_{z} ; \varepsilon_{\mathbf{k} \nu}$ is the energy for a state with Bloch vector $\mathbf{k}$, band index $\nu$, and spin index $\sigma$.

Figure 5 shows the ballistic conductances in the $\mathbf{c}$ direction for both $c=3 a_{0}$ (solid line) and $c=4 a_{0}$ (dashed line) cases. In order to indicate the contributions from different bands, we plot the conductances as a function of the energy. For the majority spin channel, around the Fermi energy the ballistic conductance for $c=3 a_{0}$ is ten times larger than the one for $c=4 a_{0}$ due to substantially larger velocity components, although the densities of states are similar for the two cases. For the minority spin channel, the ballistic conductances are zero for both cases in the vicinity of $0.3 \mathrm{eV}$ above the Fermi energy. The calculated results suggest that the $c$ $=3 a_{0}$ superlattice involving the MnAs and CrAs will be a good spintronic material.

In summary, we have examined ballistic conduction in a

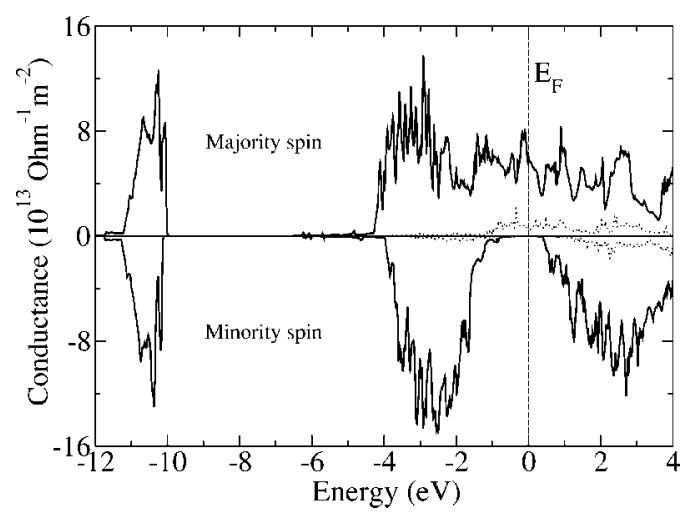

FIG. 5. Ballistic conductances along the $\mathbf{c}$ direction for the thin superlattice as a function of the energy. The Fermi energy is set to zero. The solid and dotted lines represent the $c=3 a_{0}$ and $c=4 a_{0}$ cases, respectively. 
thin superlattice composed of $\mathrm{Ga}_{0.5} \mathrm{Mn}_{0.5} \mathrm{As}, \mathrm{CrAs}$, and $\mathrm{GaAs}$ half metallic and semiconducting layers. Based on an examination of electronic structure and charge distributions, we find that upon bringing separated slabs together, a half metallic superlattice is formed with a clear conducting channel spanning the length of the superlattice; whereas, due to the presence of surface states, half metallicity is not manifested in the separated configuration. Such a half metallic superlattice grown on GaAs may be an excellent candidate for spintronic applications.
Work at UC Davis is supported by NSF Grant Nos. ESC-0255007 and INT-9872053, the Materials Research Institute at Lawrence Livermore National Laboratory (LLNL), NSERSC at Lawrence Berkeley National Laboratory, and the Research Committee at UC Davis. Work at LLNL was performed under the auspices of the U.S. Department of Energy by University of California, Lawrence Livermore National Laboratory under Contract No. W-7405Eng-48.
*Electronic address: mqian@physics.ucdavis.edu

${ }^{1}$ R. A. de Groot, F. M. Mueller, P. G. van Engen, and K. H. J. Buschow, Phys. Rev. Lett. 50, 2024 (1983).

${ }^{2}$ W. E. Pickett and J. S. Moodera, Phys. Today 54 (5), 39 (2001).

${ }^{3}$ H. Akinaga, T. Manago, and M. Shirai, Jpn. J. Appl. Phys., Part 2 39, L1118 (2000).

${ }^{4}$ J. H. Zhao, F. Matsukura, T. Takamura, E. Abe, D. Chiba, and H. Ohno, Appl. Phys. Lett. 79, 2776 (2001).

${ }^{5}$ S. Sanvito and N. A. Hill, Phys. Rev. Lett. 87, 267202 (2001); S. Sanvito, Phys. Rev. B 68, 054425 (2003).

${ }^{6}$ Y.-J. Zhao, W. T. Geng, A. J. Freeman, and B. Delley, Phys. Rev. B 65, 113202 (2002).

${ }^{7}$ I. Galanakis and P. Mavropoulos, Phys. Rev. B 67, 104417 (2003).

${ }^{8}$ J. E. Pask, L. H. Yang, C. Y. Fong, W. E. Pickett, and S. Dag, Phys. Rev. B 67, 224420 (2003).

${ }^{9}$ G. A. Prinz, Science 282, 1660 (1998).

${ }^{10}$ P. Ball, Nature (London) 404, 918 (2000).

${ }^{11}$ S. A. Wolf, D. D. Awschalom, R. A. Buhrman, J. M. Daughton, S. von Molnár, M. L. Roukes, A. Y. Chtchelkanova, and D. M. Treger, Science 294, 1488 (2001).

${ }^{12}$ C. Y. Fong, M. C. Qian, J. E. Pask, L. H. Yang, and S. Dag, Appl. Phys. Lett. 84, 239 (2004).
${ }^{13}$ R. K. Kawakami, E. Johnston-Halperin, L. F. Chen, M. Hanson, N. Guébels, J. S. Speck, A. C. Gossard, and D. D. Awschalom, Appl. Phys. Lett. 77, 2379 (2000).

${ }^{14}$ M. Mizuguchi, H. Akinaga, T. Manago, K. Ono, M. Oshima, M. Shirai, M. Yuri, H. J. Lin, H. H. Hsieh, and C. T. Chen, J. Appl. Phys. 91, 7917 (2002).

${ }^{15}$ M. C. Payne, M. P. Teter, D. C. Allen, T. A. Arias, and J. D. Joannopoulos, Rev. Mod. Phys. 64, 1045 (1992).

${ }^{16}$ P. Hohenberg and W. Kohn, Phys. Rev. 136, B864 (1964); W. Kohn L. J. Sham, Phys. Rev. 140, A1133 (1965).

${ }^{17}$ J. P. Perdew, K. Burke, and M. Ernzerhof, Phys. Rev. Lett. 77, 3865 (1996); J. P. Perdew and Y. Wang, Phys. Rev. B 46, 12947 (1992).

${ }^{18}$ VASP, Institut fur Theoretische Physik of the Technische Universitat, Wien, Austria.

${ }^{19}$ G. Kresse and J. Hafner, J. Phys.: Condens. Matter 6, 8245 (1994); G. Kresse and J. Furthmuller, Phys. Rev. B 54, 11169 (1996).

${ }^{20}$ D. Vanderbilt, Phys. Rev. B 41, R7892 (1990).

${ }^{21}$ H. J. Monkhorst and J. D. Pack, Phys. Rev. B 13, 5188 (1976).

${ }^{22}$ K. M. Schep, P. J. Kelly, and G. E. W. Bauer, Phys. Rev. B 57, 8907 (1998). 